\title{
论 文 共融机器人专题
}

\section{并联机器人执行系统的能效分析}

\author{
毕伟尧 ${ }^{1,2}$, 谢福贵 1,2 , 刘辛军 ${ }^{1,2 *}$
}

1. 清华大学机械工程系摩擦学国家重点实验室, 清华大学(机械系)-西门子先进工业机器人联合研究中心, 北京 100084 ;

2. 清华大学精密超精密制造装备及控制北京市重点实验室, 北京 100084

*E-mail: xinjunliu@mail.tsinghua.edu.cn

收稿日期: 2018-05-23; 接受日期: 2018-09-13; 网络版发表日期: 2018-11-20

国家自然科学基金(批准号: 91748205, 51675290)资助项目

\begin{abstract}
摘要 机械执行系统作为机器人等装备的关键组成部分, 其能效分析与评价在装备能效研究中具有重要意义. 当 采用并联机构时, 执行系统结构复杂, 能效分析困难. 针对这种情况, 通过分析并联机器人执行系统的能量传递机 理, 建立了执行系统能效模型和支链功率方程, 将能量从驱动单元到末端执行器的传递过程中的支链动能变化作 为影响能效的重要因素。在此基础上, 结合驱动单元能量耗散特点和末端执行器运动时支链动能变化的各向异 性, 提出了一种基于支链动能变化率的并联机器人执行系统的功率传递指标, 该指标与机构位姿和执行系统几 何、质量参数有关, 反映了并联执行系统中支链构件对能量传递的影响, 以及支链几何参数与执行系统能效之间 的关系。分别以一种二自由度平面机器人和一种五自由度空间并联机器人为例, 给出了功率传递指标的求解过 程, 分析了工作空间内机器人执行系统能效大小分布情况和高能效工作区域范围. 所提出的功率传递指标可用于 评价并联机器人执行系统的能效, 并进行并联机器人高能效执行系统的设计.
\end{abstract}

关键词能效分析, 并联机器人, 功率方程, 机械执行系统

\section{1 引言}

节能减排、提高能源利用率是当今社会关注的热 点. 一方面, 在工业界, 精益生产、绿色制造等概念相 继诞生，对制造装备的能效要求越来越高; 而另一方 面, 机器人、机床等整机装备耗能高、能量利用率低, 但节能潜力巨大 ${ }^{[1]}$. 因此, 如何降低机械装备系统的能 耗、提高能效是亟待解决的问题. 为此, 国内外学者针 对装备系统的能量特性、能耗机理以及节能方法开展 了许多研究.
现代机械装备系统由驱动单元(包括电动机、液 压马达等)、机械执行系统、控制系统及辅助器件构 成, 具有多源能耗特性, 涉及机、电、液等多学科. 能 耗分为机械损耗、电机损耗、液压系统损耗等，相应 能耗部件包括执行系统、电机、液压元件、控制装置 等 $^{[2]}$, 能耗机理与提高能效的方法各不相同. 标准ISO 14955-1中给出了机械制造系统各个部件的节能潜力 和措施 ${ }^{[3]}$.

作为机器人等机械装备系统的关键组成部分, 机 械执行系统的能效问题一直是生产者和科学研究工作

引用格式: 毕伟尧, 谢福贵, 刘辛军. 并联机器人执行系统的能效分析. 中国科学: 技术科学, 2018, 48: 1331-1340 Bi W Y, Xie F G, Liu X J. Energy efficiency analysis of parallel robot execution systems (in Chinese). Sci Sin Tech, 2018, 48: 1331-1340, doi: 10.1360/ N092018-00156 
者关心的问题. 广义上说, 机械执行系统能效是装备系 统工作能力的体现. 例如, 提高机床执行系统的转动能 力可以提高装备加工更为复杂曲面的能力, 甚至具有 五面加工的能力 ${ }^{[4,5]}$; 能够通过减少装卸工序, 避免工 序间误差引入, 同时节省停机时间, 提高了工件加工效 率和生产经济效益. 又例如, 结构紧凑、加工灵活的移 动式加工机器人改进了离线分体加工方案质量差和效 率低等问题, 在加工大尺寸工件时具有显著优势, Neugebauer等人 ${ }^{[6]}$ 探讨了此类移动式装备在提高能效上的 潜力.

狭义上，机械执行系统的能效是指执行系统的工 作效率. 工作效率是工作能力的一种，其反映了执行 系统的能量利用率 ${ }^{[7]}$. 根据研究对象的不同, 执行系统 能效分析优化分为两个层次: 整体设计和零部件优化. 在整体设计层面的能效分析优化涵盖机构构型、尺 寸、电机选型、平衡等方面. 如机床执行系统的鲁棒 性、小型化等特点对能效有重要影响, 需要分析 ${ }^{[6]}$; 在 构型设计上, 是否添加午余驱动对机器人能效有影响. 文献[8]分析了并联机器人咒余驱动对减小能耗的作 用. 当执行系统整体设计确定时, 能效优化体现在关节 润滑、部件拓扑结构优化等零部件层面. 如Kroll等人 的研究表明机床部件的轻量化设计能提高整机能效, 当机床伺服驱动系统中质量减小 $30 \%$ 时，节能高达 $50 \%{ }^{[9]}$.

无论针对何种研究对象, 建立能耗模型和评价方 法是能效分析的前提. 有些研究中, 制造装备的碳足 迹 ${ }^{[10]}$ 、释放的热能 ${ }^{[11]}$ 等被用来衡量能效. 但目前建立 能耗模型的主流方法是用与功率相关的比值，这种方 法更加直观的体现本质一能量利用率. 比值的分子是 有用功率，分母有两种不同的选取方法，代表了两类 不同研究思路. 其一是采用有用功率与总功率的比值 建模 ${ }^{[6-9]}$, 这种建模方法简化了能耗原理的研究, 适合 试验研究不同条件变化对能效的影响. 其二是实际功 率与理论最大功率(视在功率)的比值 ${ }^{[12 \sim 17]}$, 这种建模 方法揭示了执行系统内的能量传递关系. Pfefferkorn 等人 ${ }^{[12]}$ 和Tsai等人 ${ }^{[13]}$ 将单一支链中实际有用功率和理 论上最大功率之比定义为功率系数, 并提出了相关指 标. 清华大学团队研究了并联机构运动和力传递及约 束特性, 采用了能效系数的概念, 在此基础上建立了 并联机构的运动/力传递性能评价方法 ${ }^{[14 ~ 17]}$.

目前，执行系统所采用机构类型分为串联机构、
并联机构以及集成两者的混联机构 ${ }^{[18]}$. 串联机构采用 开放支链结构 ${ }^{[19]}$, 对采用串联机构的执行系统(串联机 器人、传统机床等)进行能效分析时常常将系统分解 为几个独立部分分别研究. 国内学者刘飞和施金良等 人 ${ }^{[20-22]}$ 建立了机床主传动系统的能耗模型, Altintas和 Cao利用有限元方法分析了机床主轴的能量变化规

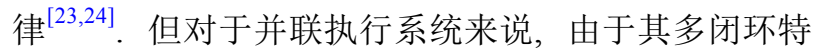
点, 能量传递机理更为复杂, 能效分析困难, 相关研究 较少. 另一方面, 在绝大多数的能效研究中, 机器人、 机床等装备系统本身被认为是黑盒子, 能耗建模是通 过实验或测量获得的，在原理上，尤其是构件尺寸设 计上, 较少有直接的讨论和相应的指标 ${ }^{[6,25]}$.

针对这些问题, 本文对并联机器人执行系统进行 能效分析，建立了并联执行系统的能量传递机理和能 效模型. 在此基础上, 通过支链功率方程, 分析并联执 行系统在末端执行器运动中的能耗损失, 提出了基于 支链动能变化率的并联执行系统的能效分析方法和能 效指标, 并结合一种二自由度平面机器人和一种五自 由度空间并联机器人的执行系统进行了分析.

\section{2 并联机器人执行系统能量传递及功率传 递指标}

\section{1 并联机器人执行系统的能量传递与能效模型}

机器人执行系统能耗建模时, 将主动关节处的驱 动单元做功及能耗作为总的输入能量, 即末端执行器 在给定负载下走过一段轨迹时, 输入总能量为驱动单 元累积耗能 $E_{\text {input }}$.

$$
E_{\text {input }}=\int_{t_{\text {start }}}^{t_{\text {stop }}[}\left[\sum_{i=0}^{i=n_{l}}\left(f_{i}(t) \cdot v_{i}(t)\right)+\sum_{i=0}^{i=n_{r}}\left(\tau_{i}(t) \cdot \omega_{i}(t)\right)\right],
$$

其中, $n_{l}, n_{r}$ 分别是直线驱动单元和转动驱动单元关节 的数目; $f_{i}(t), v_{i}(t)$ 分别为直线驱动单元关于时间的驱动 力函数和线速度函数; $\tau_{i}(t), \omega_{i}(t)$ 分别为转动驱动单元 关于时间的驱动力矩函数和角速度函数.

并联机器人执行系统有两大部分构成: 各个支链 和末端操作器(刀具、抓手等). 机械执行系统能量传 递机理需要从其结构特点上考虑. 不同于串联机器人 在结构上具有开放式特征(图1(a)), 并联机器人驱动单 元与末端执行器之间用连杆等构件形成多闭环, 驱动 单元全部布置在一端, 如图1(b)所示. 并联执行系统的 
(a)

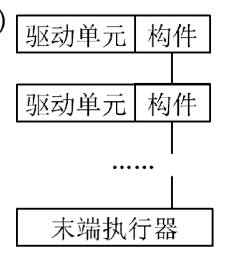

(b)

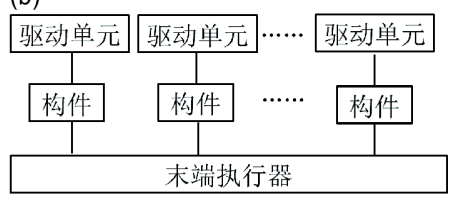

图 1 机械系统的结构特点. (a) 串联系统结构特点; (b) 并联 系统结构特点

能量从驱动单元输入, 经过执行系统的传递, 最终传递 到末端执行器上用于做功. 故而从广义上而言, 并联执 行系统支链是一种能够改变末端执行器运动形式和大 小, 向末端执行器传递能量的机械传动系统. 基于此可 以得到并联执行系统的能量传递机理, 如图2所示. 并 联执行系统的主动关节从驱动单元处获得总能量，能 量沿着各个支链的关节和杆件传递到末端执行器; 输 入总能量 $E_{\text {input }}$ 一部分用于末端执行器运动和克服外力 做功 $E_{\text {useful }}$, 一部分因关节摩擦阻尼的原因转化为热量 耗散 $E_{\text {loss, }}$, 还有一部分被改变运动状态的执行系统各支 链(传动系统)以动能和势能的形式保留下来 $E_{\text {trans }}$. 即:

$E_{\text {input }}=E_{\text {useful }}+E_{\text {loss }}+E_{\text {trans }}$.

基于并联执行系统能量传递分析，得到其能效模 型为

$\eta_{e}=E_{\text {useful }} / E_{\text {input }}$.

考虑单位时间内的能量变化，将式(3)右边分子分 母同除以单位时间转化为

$\eta_{e}=P_{\text {useful }} / P_{\text {input }}$,

其中, $\eta_{e}$ 为末端执行器做功的有用功率与输入总功率 的比值, 表示机械执行系统的功率传递效率.

\section{2 并联机器人执行系统支链的功率方程}

由 2.1 节所述可知, 制造装备机械执行系统的输入 功率等于摩擦耗散的损耗功率、用于末端做功的输出 功率以及构件自身能量的变化率三者之和。对于并联 机器人执行系统, 能量从驱动单元输入端传递到末端 执行器用于做功, 在传递过程中, 执行系统本身能量 变化, 在能量流中保留了一部分能量. Li和Bone对一 组驱动电机相同、构件尺寸和工作空间类似的串联机 器人和并联机器人进行能耗比较, 结果发现并联机器 人能耗仅为串联机器人的 $26 \%$, 其主要原因是并联机
器人移动构件的质量小 ${ }^{[26]}$. 这说明构件自身能量的变 化影响了系统能量的利用率, 这在能效分析中具有重 要意义.

需要说明的是, 2.1 节中能量传递和能效模型中考 虑了重力和摩擦, 而本文主要研究驱动单元中输入能 量的传递效率, 探讨机器人执行系统的构型、尺寸、 构件质量等因素与输入功率之间的关系, 重力势能的 变化与机器人放置方式等外在因素有关. 为体现执行 系统对输入能量传递的影响, 文中分析及指标定义不 考虑重力因素, 同时忽略支链的关节摩擦, 设关节上 的力是非耗散力.

图3展示了并联执行系统中支链构件的受力分析 图, 为了使表达更清晰简便, 图3中运动和力用运动旋 量和力旋量表示. $\$_{I k} 、 \$_{T k}$ 和 $\$_{O k}$ 分别表示第 $k$ 个支链的 驱动关节运动旋量、支链作用于末端执行器上的力旋 量和末端执行器运动旋量; $\$_{j}$ 表示第 $j$ 个关节上的力旋 量, $j=1 \sim m, m$ 为支链关节数量; $\$_{i}$ 表示第 $i$ 个构件的运 动旋量. 驱动关节运动旋量表示驱动关节对应的运动 旋量. 支链作用于末端执行器上的力旋量将驱动关节 的运动和力传递到末端执行器. 末端执行器运动旋

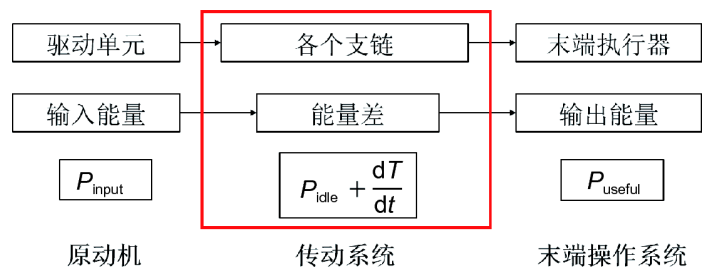

图 2 (网络版彩图) 并联机器人执行系统的传动系统等效 原理

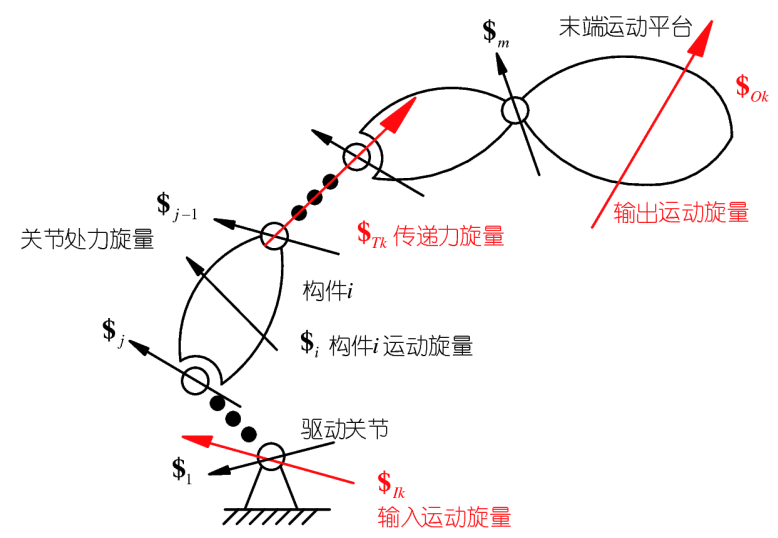

图 3 (网络版彩图)并联执行系统中支链构件的受力分析 
量 $\mathbf{\$}_{O k}$ 表示末端执行器的瞬时运动.

根据旋量理论，力旋量与运动旋量的互易积表示 两者之间的功率. 支链作用于末端执行器上的力旋 量 $\mathbf{\$}_{T k}$ 对末端执行器运动旋量 $\mathbf{S}_{O k}$ 做功表示支链传递到 末端执行器的能量, 瞬时功率可用两者互易积表示.

以第 $k$ 支链的第 $i$ 个构件为例进行分析，该构件运 动用运动旋量 $\mathbf{S}_{i}$ 表示, 在前一级构件连接处受力旋量 $\mathbf{\$}$ 作用, 在下一级构件连接处受力旋量 $\mathbf{S}_{j+1}$ 作用.

$\mathbf{\$}_{i}=\left(\boldsymbol{\omega}_{i} ; \mathbf{v}_{i}\right)$,

$\mathbf{\$}_{j}=\left(\mathbf{f}_{j} ; \boldsymbol{\tau}_{j}\right)$,

$\boldsymbol{\$}_{j+1}=\left(\mathbf{f}_{j+1} ; \boldsymbol{\tau}_{j+1}\right)$.

由螺旋理论基本概念可知，力旋量和运动旋量的 互易积表示力旋量对按此运动旋量运动下物体所做功 的瞬时功率, 即可以表示成无穷小的元功. 第 $i$ 个构件 两端受力所做的元功表示为式(8)和(9).

$\delta W_{1}=\boldsymbol{\$}_{i} \circ \boldsymbol{\$}_{j}=\mathbf{v}_{i} \cdot \mathbf{f}_{j}+\boldsymbol{\omega}_{i} \cdot \boldsymbol{\tau}_{j}$.

$\delta W_{2}=\boldsymbol{\$}_{j+1} \circ \boldsymbol{\$}_{i}=\mathbf{f}_{j+1} \cdot \mathbf{v}_{i}+\boldsymbol{\tau}_{j+1} \cdot \boldsymbol{\omega}_{i}$.

由质点系动能定理, 刚体上所有力的元功之和等 于刚体动能的微分. 故支链中构件动能的微分表示系 统能量传递时构件在外力作用下的能量变化.

$\mathrm{d} T=\sum_{i=1}^{n} \delta W_{i}=\delta W$.

公式(10)推广至整个支链可得

$\mathrm{d} T=\sum_{i=1}^{n} \delta W_{i}=\delta W_{I}+\delta W_{b 1 b 2}+\delta W_{b 2 b 1}+\cdots+\delta W_{j n O}$,

其中, $\delta W_{I}$ 表示输入力对支链做的元功, $\delta W_{O}$ '表示负载 对支链做的功, $\delta W_{b 1 b 2}$ 表示支链中刚体 $b 1$ 对刚体 $b 2$ 所做 的元功, 以此类推. 支链内部各关节上的力为内力, 正 负相消, 不会改变支链总能量, 即 $\delta W_{b 1 b 2}+\delta W_{b 2 b 1}=0$. 而 负载对支链所作的元功 $\delta W_{O}{ }^{\prime}$ 与支链对负载所做的元 功 $\delta W_{O}$ 之间互为相反数, 故式(11)变为

$\mathrm{d} T=\sum_{i=1}^{n} \delta W_{i}=\delta W_{I}+\delta W_{O}{ }^{\prime}=\delta W_{I}+\delta W_{O}$.

由于元功表示的是 $\mathrm{d} t$ 时间内做的功 $\delta W=\mathrm{d} t \cdot P$, 式 (12)左右同除 $\mathrm{d} t$, 且力旋量和运动旋量的互易积可以表 示力对此运动物体做功的瞬时功率，同时注意到动能
变化率的单位与功率单位相同, 将 $\mathrm{d} T_{k} / \mathrm{d} t$ 定义为支链传 递功率 $P_{\text {trans }}, P_{\text {trans }}$ 体现了能量从输入到输出传递过程 中支链的截留部分, 得到第 $k$ 个支链的功率方程为

$\mathrm{d} T_{k} / \mathrm{d} t=P_{\text {trans }}=P_{I k}-P_{O k}=\mathbf{\$}_{T k} \circ \mathbf{\$}_{I k}-\mathbf{\$}_{T k} \circ \mathbf{\$}_{O k}$.

当支链动能变化率 $\mathrm{d} T_{k} / \mathrm{d} t$ 大于 0 时, 意味着在驱动 单元和末端执行器之间传递能量的杆件动能增加; 而 当 $\mathrm{d} T_{k} / \mathrm{d} t$ 值为负时, 表示杆件能量减少, 杆件向外做功. 杆件向外做功的对象可以是末端执行器, 也可以是驱 动单元. 而实际上杆件传递到驱动单元的能量被驱动 单元阻尼器变成了热损失.

支链在运动过程中的能量来源于驱动电机, 故从 能量利用率角度出发, 支链能量可视作能量从输入端 到输出端的截留部分, 支链截留能力与机构尺寸、支 链自身质量大小及分布等有关. 通常希望驱动单元的 输入功率能全部转化为末端输出功率. 支链截留能力 的容积越小越好, 所以支链运动过程中, 构件在运动 中的动能变化率 $\mathrm{d} T / \mathrm{d} t$ 越小越好.

\section{3 并联机器人执行系统的功率传递评价指标}

基于 2.2 节中的概念，本节定义并联执行系统的能 效评价指标. 机器人执行系统的能效与诸多因素有关, 包括: 机械执行系统的机构构型、尺寸、质量、外加 负载和末端执行器的运动状态. 本文研究的重点是机 械执行系统自身特性对系统能效的影响. 在评价方法 上，为了体现执行系统在能量传递中的作用，考虑当 末端执行器匀速运动且不受外界作用力的情况. 此时, 末端执行器动能恒定, 既不输出功率, 也不接受功率输 入, 避免了末端执行器质量和转动惯量对能效分析的 影响，进而集中探讨执行系统机构构型、杆件质量与 尺寸对能效传递的影响. 且由于末端执行器匀速运动, 外载的变化不会影响指标值. 同时, 当设外界作用力为 零时, 机器人对外不做功, 驱动单元输入能量仅用于改 变支链的运动状态, 支链动能变化率直接与驱动单元 输入功率相等, 即指标值越小(大), 输入功率越小(大), 能效越高(低). 在末端执行器速度选取上, 考虑到并联 执行系统动能变化在不同末端执行器速度方向上的各 向异性, 为了整体评价机构的能效, 需考察并联装备的 末端执行器在向不同方向以单位速度匀速运动时(自 由度允许)的能效, 即末端执行器速度选取采用并联机 构的许动运动空间单位基底旋量及其反方向的单位基 
底旋量, 其中, 许动运动旋量表示机构在自由度方向上 允许发生的运动，许动运动子空间定义为机构系统中 所有线性无关的许动运动旋量张成的 $n(0 \leq n \leq 6)$ 维子 空间.

支链的动能变化率与支链中刚体的质量和转动惯 量有关, 也与刚体的运动状态有关. 对支链刚体进行轻 量化设计可以通过减小质量来减少 $P_{\text {trans. }}$ 除质量外, 刚 体的运动状态由机构的运动学求得, 与机构尺寸有关, 所以另一个重要影响因素是机构尺寸. 为讨论并联执 行系统支链变化率与并联机构尺寸的关系，执行系统 各刚体动能变化率除以相应质量, 可得末端执行器在 某一运动方向上以单位速度匀速运动时并联执行系统 的功率传递指标，根据2.2节中支链能量释放特点，指 标定义为

$$
\lambda_{q}=\left[\sum_{i=1}^{n} \frac{\mathrm{d} T_{i q} / \mathrm{d} t}{m_{i}}\right]^{+},
$$

其中, 下标 $q$ 表示末端执行器匀速运动的方向为第 $q$ 个 运动方向; $i$ 表示第 $i$ 个刚体， $i=1,2, \ldots, n$ ，其质量为 $m_{i}$; 符号 $[a]^{+}$表示如果 $a$ 的值大于等于零, $[a]^{+}$的值为 $a$, 如 果 $a$ 的值小于零, $[a]^{+}$的值为零. 指标物理意义为当末 端执行器在某方向以单位速度匀速运动时, 并联机器 人执行系统各杆件中的所有刚体的单位质量动能变化 率之和, 变化范围为 $[0,+\infty)$.

为了整体评价执行系统的功率传递性能, 需考虑 不同末端速度方向对能效的影响，定义并联执行系统 的功率传递指标为

$\eta=\max _{q}\left\{\lambda_{q}\right\},(q=1,2, \ldots, m)$,

其中, $m$ 是并联机构的许动运动空间单位基底旋量数 目之和的两倍.

指标 $\eta$ 描述了在给定姿态和并联执行系统几何参 数下, 并联执行系统的传递功率大小, $\eta$ 值越小, 表示并 联执行系统从输入端到输出端的功率传递效率越高, 即能效越高.

\section{3 案例分析}

\section{1 案例 1: 二自由度平面并联机器人}

\section{1 .1 二自由度平面并联机器人介绍}

下面以二自由度平面 $5 \mathrm{R}$ 并联机器人为例, 给出指
标的求解过程和说明.

如图4所示, 全局坐标系 $o-x y$ 的原点位于转动副 $A$ 和 $E$ 的中点. 转动副 $A$ 和 $E$ 为驱动关节, $A$ 和 $E$ 的中心点 到原点 $O$ 的距离为 $r_{1}$, 驱动杆 $A B$ 和 $E D$ 的杆长均为 $r_{2}$, 而 随动杆 $B C$ 和 $D C$ 的杆长均为 $r_{3}$.

\subsection{2 机构微分运动学分析}

若要求并联执行系统中各个杆件的动能变化率, 首先需要对该执行系统采用的并联机构进行微分运动 学分析, 求出各个杆件的速度、加速度、角速度和角 加速度. 求解过程介绍如下.

设末端执行器的位置为 $(x y), \theta_{1}, \theta_{2}, \theta_{3}, \theta_{4}$ 分别为 杆 $A B, B C, E D, D C$ 与水平面的夹角, 先求杆 $A B$ 和 $B C$ 的 微分运动学解, 在 $x$ 轴和 $y$ 轴投影可得

$\left\{\begin{array}{c}r_{2} \cos \theta_{1}+r_{3} \cos \theta_{2}-r_{1}=x \\ r_{2} \sin \theta_{1}+r_{3} \sin \theta_{2}=y\end{array}\right.$

利用 $\sin ^{2} \theta_{1}+\cos ^{2} \theta_{1}=1$ 可消去 $\theta_{1}$, 求解出 $\theta_{2}$, 代入可 解得 $\theta_{1}$. 各杆质心位置可由 $\theta_{1}, \theta_{2}$ 求得.

求解速度时, 将式(16)对时间求导, 可得

$\left\{\begin{array}{l}-r_{2} \dot{\theta}_{1} \sin \theta_{1}-r_{3} \dot{\theta}_{2} \sin \theta_{2}=\dot{x}, \\ r_{2} \dot{\theta}_{1} \cos \theta_{1}+r_{3} \dot{\theta}_{2} \cos \theta_{2}=\dot{y} .\end{array}\right.$

可得到 $\dot{\theta}_{1}, \dot{\theta}_{2}$, 进而可得杆件 $A B$ 和 $B C$ 的角速度和质 心速度.

求解加速度时, 将式(17)对时间求导, 可得

$\left\{\begin{array}{l}-r_{2} \dot{\theta}_{1}^{2} \cos \theta_{1}-r_{2} \ddot{\theta}_{1} \sin \theta_{1}-r_{3} \ddot{\theta}_{2} \sin \theta_{2}-r_{3} \dot{\theta}_{2}^{2} \cos \theta_{2}=\ddot{x}, \\ r_{2} \ddot{\theta}_{1} \cos \theta_{1}-r_{2} \dot{\theta}_{1}^{2} \sin \theta_{1}+r_{3} \ddot{\theta}_{2} \cos \theta_{2}-r_{3} \dot{\theta}_{2}^{2} \cos \theta_{2}=\ddot{y} .\end{array}\right.$

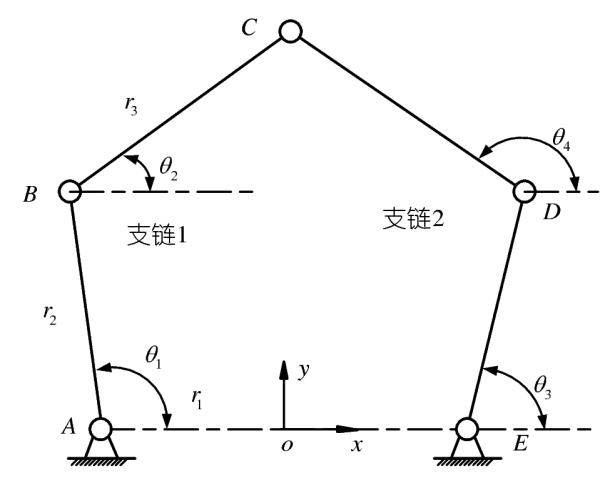

图 4 平面5R并联机构运动简图 
可得到 $\ddot{\theta}_{1}, \ddot{\theta}_{2}$, 进而可得杆件 $A B$ 和 $B C$ 的角速度和质 心加速度.

同理可得杆件 $E D$ 和 $D C$ 的质心位置、速度、加速 度、角速度和角加速度. 为指标求解打下基础.

\subsection{3 功率传递指标求解}

根据柯尼希定理, 并联机器人执行系统杆件的总 动能为

$$
T=\sum_{i=1}^{4}\left(\frac{1}{2} m_{i}\left|\mathbf{v}_{i C}\right|^{2}+\frac{1}{2} \boldsymbol{\omega}_{i}{ }^{T} \cdot \mathbf{J}_{i C} \cdot \boldsymbol{\omega}_{i}\right),
$$

其中, $m_{i}$ 表示杆件中刚体 $i$ 的质量, $\mathbf{v}_{i C}$ 表示刚体 $i$ 的质心 速度, $\mathbf{J}_{i C}$ 表示刚体 $i$ 绕质心坐标系的转动惯量, $\boldsymbol{\omega}_{i}$ 表示 刚体 $i$ 的角速度.

上式求导可得

$\mathrm{d} T_{i} / \mathrm{d} t=m_{i} v_{C i} a_{C i}+J_{C i} \omega_{i} \varepsilon_{i}$.

设机构几何参数为 $r_{1}=0.55 \mathrm{~m}, r_{2}=0.85 \mathrm{~m}, r_{3}=1.6 \mathrm{~m}$, 求解在机器人全部工作空间内的功率传递指标值 $\eta$, 结 果如图5所示. 指标值越小表示末端执行器在工作空间 内此处以单位运动匀速运动时, 支链的动能变化率越 小, 能效越高. 从图中可看出, 在接近理论工作空间边 界, 即机构的边界奇异轨迹时, 功率传递指标会急剧变 大，而远离边界奇异轨迹时，功率传递指标值会减小; 在工作空间内部, 指标值小且没有出现集聚, 指标分布 不但可以表示能效大小，也可以很好的评价机构距离 边界奇异位置的远近.

\section{2 案例2: 五自由度空间并联机器人}

\subsection{1 五自由度空间并联机器人介绍}

下面以一种五自由度并联机器人为例, 对上述指 标进行说明.

图6为并联装备的执行系统CAD模型和机构运动 简图，其运动学逆解见文献[5]. 机构主轴和基座之间 由三条支链连接. 第一条支链 $B_{1} P_{1}$ 可称作URPR或 $\mathrm{SPR}$ 运动支链 $(U$, 虎克铰; $R$, 转动副; $P$, 移动副, 下划线表 示主动驱动; $\mathrm{S}$, 球铰), 另外两条支链结构相同且关节 组成顺序为R-2RP-R-S. 根据其关节组成，可表示为 SPR-2(R-2RP-R-S)机构。整个机构有五个自由度，五 个主动移动副驱动, 动平台的位姿可以用 $(x, y, z, \varphi, \theta)$ 表示. 其中, $(x, y, z)$ 表示点 $o^{\prime}$ 在绝对坐标系 $R: o-x y z$ 中

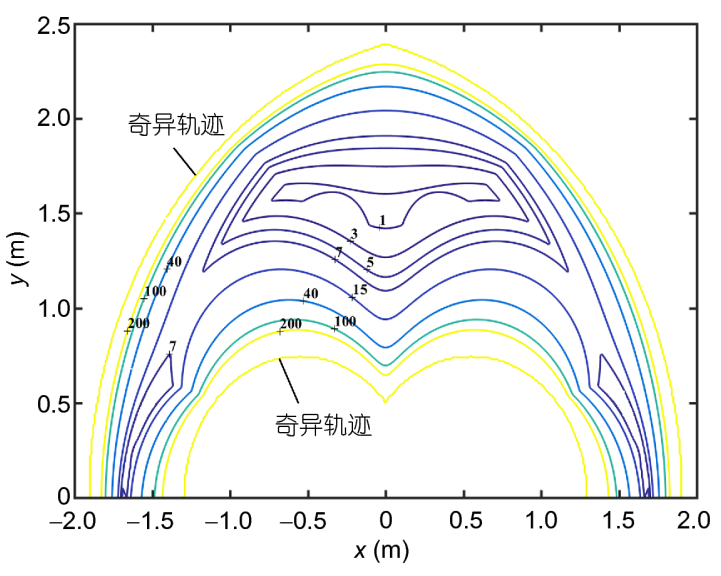

图 5 (网络版彩图)功率传递指标在理论工作空间内的分布 曲线

(a)
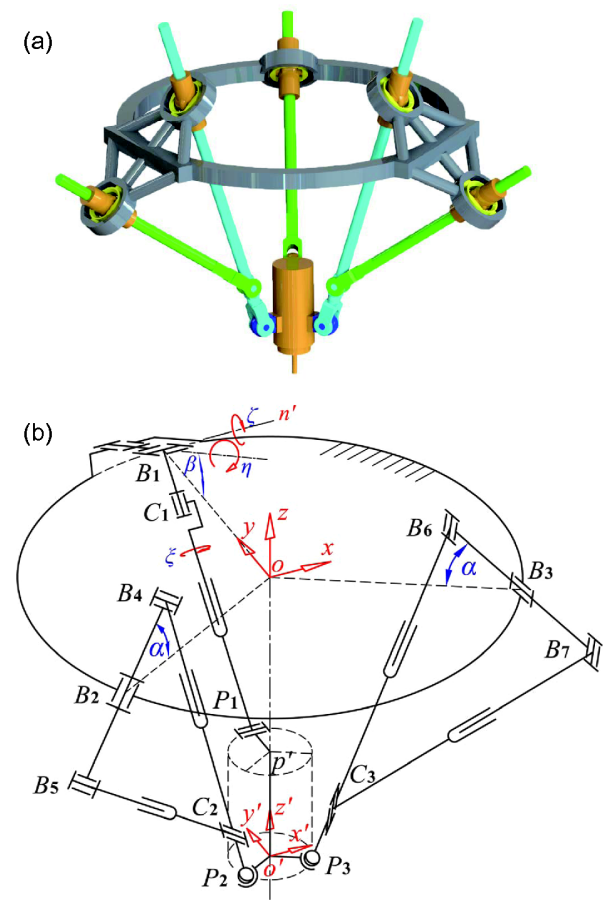

图 6 (网络版彩图)并联机器人执行系统. (a) CAD模型; (b) 机构运动简图

的坐标, $(\varphi, \theta)$ 表示 $\mathbf{o}^{\prime} \mathbf{p}^{\prime}$ 在 $\mathrm{T} \& \mathrm{~T}$ 角描述下的方向. $\varphi$ 为方 位角, $\varphi \in[0,2 \pi) ; \theta$ 为倾斜角, $\theta \in[0, \pi)$, 如图7所示.

图6(b)中的绝对坐标系 $o-x y z$ 定义如下: 平面 $o-x y$ 与平面 $B_{1} B_{2} B_{3}$ 平行, 原点 $o$ 与 $B_{1}, B_{2}$ 和 $B_{3}$ 所确定的圆的 圆心重合， $y$ 轴通过 $B_{1}$ 点. 在原点处建立局部坐标 系 $\mathfrak{R}^{\prime}: O^{\prime}-x^{\prime} y^{\prime} z^{\prime}$, 局部坐标系 $z^{\prime}$ 轴与主轴轴线重合, 当处 于初始位置时 $\left(\varphi=270^{\circ}, \theta=0^{\circ}\right), z^{\prime}$ 轴与绝对坐标系 $z$ 轴 


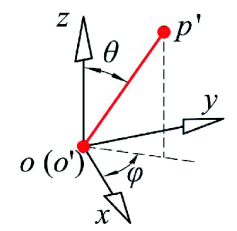

图 7 (网络版彩图)方位角 $\varphi$ 和倾斜角 $\theta$ 的定义

共线, $x^{\prime}$ 轴和 $y^{\prime}$ 轴分别与绝对坐标系中的 $x$ 轴和 $y$ 轴平 行. $P_{2}$ 和 $P_{3}$ 在局部系平面 $o^{\prime}-x^{\prime} y^{\prime}$ 内. 机构的尺寸参数定 义如下:

$\left|\mathbf{o}^{\prime} \mathbf{P}_{2}\right|=\left|\mathbf{o}^{\prime} \mathbf{P}_{3}\right|=\left|\mathbf{p}^{\prime} \mathbf{P}_{1}\right|=R_{2}$,

$\left|\mathbf{B}_{2} \mathbf{B}_{4}\right|=\left|\mathbf{B}_{2} \mathbf{B}_{5}\right|=\left|\mathbf{B}_{3} \mathbf{B}_{6}\right|=\left|\mathbf{B}_{3} \mathbf{B}_{7}\right|=W$,

$\left|\mathbf{o B}_{\mathbf{i}}\right|=R_{1}, \quad\left|\mathbf{o}^{\prime} \mathbf{p}^{\prime}\right|=L \quad(i=1,2,3)$.

$\mathbf{B}_{4} \mathbf{B}_{5}$ 和 $\mathbf{B}_{6} \mathbf{B}_{7}$ 相对于水平面 $o-x y$ 的倾斜角用 $\alpha$ 表示, 即 $\angle o B_{2} B_{4}=\angle o B_{3} B_{6}=\alpha, \alpha \in(0, \pi / 2)$. 平面 $o B_{4} B_{5}$ 和 $o B_{6} B_{7}$ 的交线与 $z$ 轴共线.

\subsection{2 机构微分运动学分析}

下面介绍各个杆件的速度、加速度、角速度和角 加速度的求解过程.

设杆 $B_{1} P_{1}, B_{4} P_{2}, B_{6} P_{3}$ 分别为 $l_{1}, l_{2}, l_{3}$, 杆 $B_{5} C_{2}, B_{7} C_{3}$ 分别为 $l_{4}, l_{5}$, 主轴局部坐标系 $\mathfrak{R}^{\prime}$ 原点 $o^{\prime}$ 在绝对坐标系 $O-$ $x y z$ 下的坐标为 $\left[\begin{array}{lll}x & y & z\end{array}\right]^{\mathrm{T}}$.

以杆 $B_{1} P_{1}$ 为例, 由矢量闭环法可得

$o B_{1}+B_{1} P_{1}^{\prime}+P_{1} o^{\prime}=\sigma^{\prime}$.

式(21)两边同时求导, 可得

$\mathbf{i}_{1}+\boldsymbol{\omega}_{1} \times \mathbf{l}_{1}+{ }^{0} \boldsymbol{\omega}_{0} \times{ }^{0} \mathbf{L}_{0}=\mathbf{v}_{p}$,

其中, $\dot{i}_{1}, \boldsymbol{\omega}_{1}$ 表示绝对坐标系下杆的平动速度和转动角 速度, ${ }^{0} \boldsymbol{\omega}_{0},{ }^{0} \mathbf{L}_{0}$ 表示主轴局部坐标系中的定点转动角速 度和 $P_{1}$ 坐标, $\mathbf{v}_{p}=\left[\begin{array}{lll}\dot{x} & \dot{y} & \dot{z}\end{array}\right]^{\mathrm{T}}$ 是主轴末端的速度矢量.

分别用 $\mathbf{I}_{1} /\left|\mathbf{I}_{1}\right|$ 点积和叉积式(22)两端, 得到杆的平 动速率和转动角速度.

$$
\begin{aligned}
& i_{1}=\mathbf{I}_{1} /\left|\mathbf{I}_{1}\right| \cdot \mathbf{v}_{p}-{ }^{0} \boldsymbol{\omega}_{0} \cdot\left({ }^{0} \mathbf{L}_{0} \times \mathbf{I}_{1} /\left|\mathbf{I}_{1}\right|\right) . \\
& \omega_{1}=\mathbf{l}_{1} /\left|\mathbf{I}_{1}\right| \times\left({ }^{0} \boldsymbol{\omega}_{0} \times{ }^{0} \mathbf{L}_{0}+\mathbf{v}_{p}\right) /\left|\mathbf{I}_{1}\right| .
\end{aligned}
$$

式(15)两端同时求导, 可得

$$
\begin{aligned}
& \ddot{\mathbf{i}}_{1}+\boldsymbol{\varepsilon}_{1} \times \mathbf{l}_{1}+\left|\boldsymbol{\omega}_{1}\right|^{2} \times \mathbf{l}_{1}+2 \boldsymbol{\omega}_{1} \times \mathbf{\mathbf { i }}_{1}+ \\
& { }^{0} \boldsymbol{\varepsilon}_{0} \times{ }^{0} \mathbf{L}_{0}+{ }^{0} \boldsymbol{\omega}_{0} \times\left({ }^{0} \boldsymbol{\omega}_{0} \times{ }^{0} \mathbf{L}_{0}\right)=\dot{\mathbf{v}}_{p},
\end{aligned}
$$

其中, $\ddot{\mathbf{i}}_{1}, \boldsymbol{\varepsilon}_{1}$ 分别表示绝对坐标系下杆 1 的平动加速度和 转动角加速度, ${ }^{0} \boldsymbol{\varepsilon}_{0}$ 主轴局部坐标系中的定点转动角加 速度, $\dot{\mathbf{v}}_{p}=\left[\begin{array}{lll}\ddot{x} & \ddot{y} & \ddot{z}\end{array}\right]^{\mathrm{T}}$ 是主轴末端的加速度矢量.

类似的, 用 $\mathbf{I}_{1} /\left|\mathbf{I}_{1}\right|$ 分别点积和叉积式(25)两端, 可以 得到杆的平动加速度和角加速度的模长.

对于杆 $B_{5} C_{2}$, 由矢量闭环法可得

$\overrightarrow{O B_{5}}+B_{5} C_{2}+C_{2} P_{2}+P_{2} O^{\prime}=\overrightarrow{o o^{\prime}}$.

注意到

$C_{2} P_{2}=\overline{o B_{4}}+B_{4} P_{2}-\left(\overline{o B_{4}}+B_{4} C_{2}\right)$.

采用与杆 $B_{1} P_{1}$ 求解时相同的步骤, 可得杆 $B_{5} C_{2}$ 的 加速度和角加速度. 杆 $B_{7} C_{3}$ 的求法类似.

这样就得到了全部 5 根杆的速度、加速度、角速 度和加角速度, 为动能变化率的求解打下基础.

\subsection{3 功率传递指标求解}

设机构尺寸为 $\alpha=41.8^{\circ}, \mathrm{L}=292.5 \mathrm{~mm}, \mathrm{~W}=542 \mathrm{~mm}$, $R_{1}=877.5 \mathrm{~mm}, R_{2}=180 \mathrm{~mm},\left|P_{2} C_{2}\right|=\left|P_{3} C_{3}\right|=200 \mathrm{~mm}$, 设 五根杆的杆长均为 $1500 \mathrm{~mm}$.

研究末端执行器在工作空间 $x \in(-400,400) \mathrm{mm}$, $y \in(-400,400) \mathrm{mm}, \mathrm{z}=-900 \mathrm{~mm}, \varphi=270^{\circ}, \theta=0^{\circ}$ 范围内 末端执行器向各个方向以单位速度运动(加速度为零) 时的机器人执行系统功率传递指标. 末端执行器的末 端状态分别取描述机构自由度的方向 $(x, y, z, \varphi, \theta)$ 及 其反方向, 根据式(15), 取最大值得到的结果如图8(a). 图8(b)为分布在设定工作空间内的等高线投影. 由于 机构沿 $y$ 轴对称, 所以图像也具有 $y$ 轴对称性. 图中点 的数值越小, 则当机器人末端执行器以单位速度运动 时, 支链动能变化率越小, 支链消耗的功率 $P_{\text {trans }}$ 越小, 输入总能量传递到输出端的越多, 能效更高. 由图中 可以看出, 并联执行系统功率传递指标沿 $y$ 轴对称, 且 $y$ 轴两侧, 指标随偏离 $y$ 轴程度变大而增长. 当 $x=0$ 时, 指 标最小值出现在 $y \in(100,200) \mathrm{mm}$ 范围内. 另外, 当 $x \in(-100,100) \mathrm{mm}, y \in(0,300) \mathrm{mm}$ 时, 功率传递 指标 $\eta<1.1$, 此区域能效值小于其他区域，即此区域可 

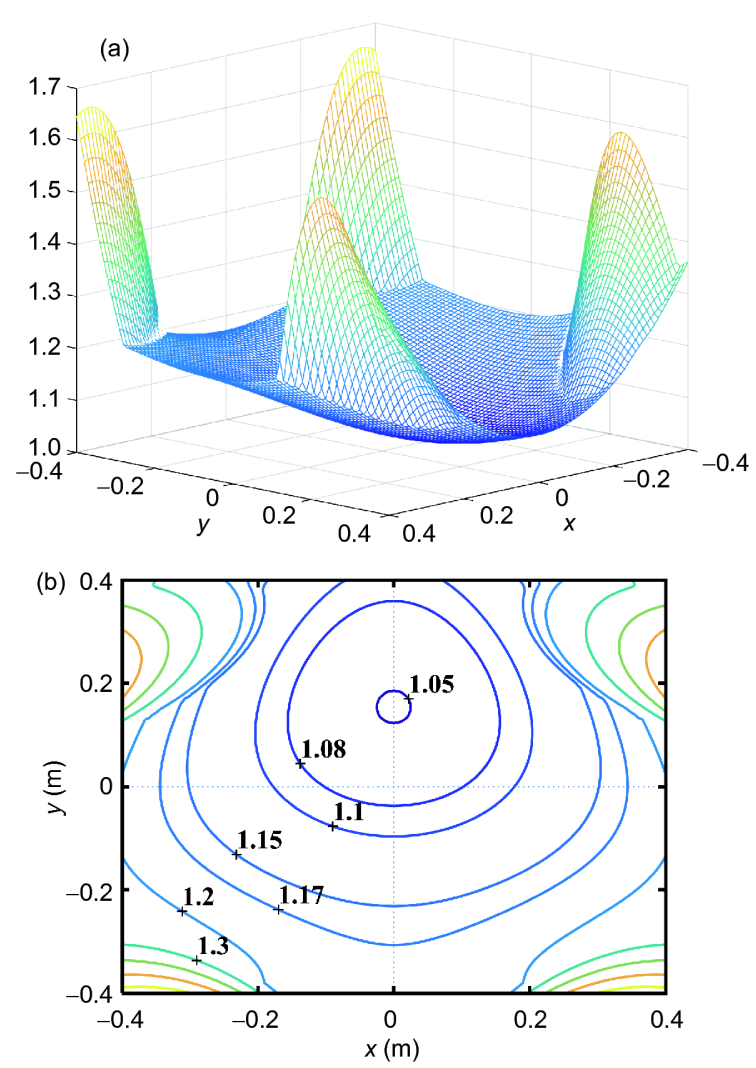

图 8 (网络版彩图) 空间并联机器人在工作空间内 $\eta$ 的分布. (a) 三维分布图; (b) 等高线图

称为工作空间内的高能效工作区域. 末端在此区域运 动时, 执行系统支链动能变化率小, 功率传递效率高, 能量利用率高.

该指标体现了并联系统执行系统的功率传递效 率, 可评价执行系统的能效. 该指标大小与执行系统的 构型以及几何参数有关, 可用于机器人高能效工作空 间的选取和指导具有高能效执行系统的并联机器人的 设计.
需要注意的是, 功率传递指标 $\eta$ 表示末端执行器在 许动运动空间内的整体评价, 即在某姿态下, 对能效的 影响最大的方向会显示在功率传递指标中, 而其他末 端运动方向不会在指标中出现. 在指标实际求解时可 以根据机器人工作条件实际工况而选择需考虑的末端 运动方向.

\section{4 结论}

根据并联机器人的结构特点, 将驱动单元之后的 机械执行系统分为用于传动的支链部分和末端执行 器, 并建立了执行系统能量传递模型. 基于能量传递模 型, 发现能量在传递过程中, 除有用功外, 输入总能量 的一部分用于改变支链运动状态, 转化为支链机械能, 支链机械能在释放时, 一部分通过驱动单元阻尼器摩 擦耗散, 并不能全部转化为有用功. 在执行系统能量 传递中, 希望来自驱动单元的能量全部传递到末端做 有用功, 故希望通过设计, 减少能量传递时执行系统 的能量消耗.

通过推导支链单位时间内的能量变化，即并联支 链的功率方程, 将能量传递过程中全部支链构件的动 能变化率作为并联执行系统能效分析的重要参考. 结 合驱动单元能量耗散特点和末端执行器运动时支链动 能变化的各向异性, 建立了机器人执行系统的功率传 递评价指标, 该指标反映了支链对执行系统能量传递 的影响和机构设计尺寸与能效之间的关系, 可用于并 联机器人执行系统的能效评价, 为具有高能效执行系 统的机器人设计提供参考. 以一个五自由度并联机器 人的执行系统为例, 说明指标求解过程, 分析了工作 空间内能效指标的分布, 并辨识得到了机器人的高能 效工作空间.

\section{参考文献}

1 刘飞, 王秋莲. 机械制造系统能效评价的特点、研究现状及发展趋势. 中国机械工程, 2013, 24: 1550-1556

2 胡韶华. 现代数控机床多源能耗特性研究. 博士学位论文. 重庆: 重庆大学, 2012

3 ISO. ISO/WD 14955-1 Environmental evaluation of machine tools-Part 1: Energy-saving design methodology for machine tools. International Organization for Standardization, 2010

4 黄真, 李秦川. 少自由度并联机器人机构的型综合原理. 中国科学E辑: 技术科学, 2009, 33: 813-819

5 Xie F G, Liu X J, Luo X et al. Mobility, singularity, and kinematics analyses of a novel spatial parallel mechanism, J Mech Robot, 2016, 8: 061022-061022-10

6 Neugebauer R, Wabner M, Rentzsch H, et al. Structure principles of energy efficient machine tools. CIRP J Manufacturing Sci Tech, 2011, 4: 
136-147

7 Carabin G, Wehrle E, Vidoni R. A review on energy-saving optimization methods for robotic and automatic Systems. Robotics, 2017,6 : 39

8 Lee G, Park S, Lee D H, et al. Minimizing energy consumption of parallel mechanisms via redundant actuation. Trans Mechatronics, 2015, 20: $2805-2812$

9 King M D, Menzel W P, Grant P S, et al. Airborne scanning spectrometer for remote sensing of cloud, aerosol, water vapor, and surface properties. J Atmos Ocean Technol, 1996, 13: 777-794

10 Kroll L, Blau P, Wabner M, et al. Lightweight components for energy-efficient machine tools. CIRP J Manufacturing Sci Tech, 2011, 4: 148-160

11 Jeswiet J, Kara S. Carbon emission and $\mathrm{CEM}^{\mathrm{TM}}$ in manufacturing. CIRP ANN Manuf Tech, 2008, 57: 17-20

12 Pfefferkorn F E, Lei S, Jeon Y, et al. A metric for defining the energy efficiency of thermally assisted machining. Int J Mach Tool Manuf, 2009, 49: 746-756

13 Tsai M J, Lee H W. Generalized evaluation for the transmission performance of mechanisms. Mechanism Machine Theor, 1994, 29: 607-618

14 Chen C, Angeles J. Generalized transmission index and transmission quality for spatial linkages. Mechanism Machine Theor, 2007, 42: 12251237

15 Wang J, Wu C, Liu X J. Performance evaluation of parallel manipulators: Motion/force transmissibility and its index. Mechanism Machine Theor, 2010, 45: 1462-1476

16 吴超. 并联机构运动和力传递特性分析及应用研究. 博士学位论文. 北京: 清华大学, 2012

17 陈祥, 谢福贵, 刘辛军. 并联机构中运动/力传递功率最大值的评价. 机械工程学报, 2014, 50: 1-9

18 陈祥. 并联机构的传递、约束和输出特性评价及其应用研究. 博士学位论文. 北京: 清华大学, 2015

19 吴元庆, 丁汉. 并联机构的等价关系和偏序关系在机构综合与自由度分析中的应用. 科学通报, 2009, 54: 3628-3691

20 曾达幸, 黄真. 基于螺旋理论的转动解耦并联机构型综合. 中国科学: 技术科学, 2011, 41: 585-891

21 刘飞, 徐宗俊, 但斌. 机械加工系统能量特性及其应用. 北京: 机械工业出版社, 1995

22 施金良, 刘飞, 许弟建, 等. 变频调速数控机床主传动系统的功率平衡方程. 机械工程学报, 2010, 46: 118-123

23 Altintas Y, Cao Y. Virtual design and optimization of machine tool spindles. CIRP ANN Manuf Tech, 2005, 54: 379-382

24 Cao Y, Altintas Y. Modeling of spindle-bearing and machine tool system for virtual simulation of milling operations. Int J Mach Tool Manuf, 2007, 47: 1342-1350

25 Bi Z M, Wang L H. Optimization of machining processes from the perspective of energy consumption: A case study. J Manuf Syst, 2012, 31: $420-428$

26 Li Y, Bone G M. Are parallel manipulators more energy efficient? In: 2001 IEEE International Symposium on Computational Intelligence in Robotics and Automation. IEEE, 2001. 8: 41-46 


\title{
Energy efficiency analysis of parallel robot execution systems
}

\author{
BI WeiYao ${ }^{1,2}$, XIE FuGui ${ }^{1,2} \&$ LIU XinJun ${ }^{1,2}$ \\ ${ }^{1}$ The State Key Laboratory of Tribology \& Tsinghua University (DME)-Siemens Joint Research Center for Advanced Robotics, Department of \\ Mechanical Engineering (DME), Tsinghua University, Beijing 100084, China; \\ ${ }^{2}$ Beijing Key Lab of Precision/Ultra-precision Manufacturing Equipments and Control, Tsinghua University, Beijing 100084, China
}

Mechanical execution systems are key components of manufacturing equipments such as robots, and energy efficiency analysis of execution systems has great significance for energy efficiency research. Since the structure of execution system adopting parallel mechanism is more complicated than its serial counterpart, the corresponding energy efficiency analysis is more difficult. By analyzing the energy transmission mechanism of parallel robot execution systems, the energy efficiency model of the execution systems is established and the power equation of limbs is derived. Then the rate of limb kinetic energy during the energy transfer from prime motors to the end effector is defined as a key factor affecting energy efficiency. On this basis, with the motor energy dissipation characteristics and the anisotropy of the kinetic energy rate, a power transmission index of parallel robot execution systems is proposed. This index is related to the mechanism pose and geometry parameters. Taking a two-degree-of-freedom planar parallel robot and a five-degree-of-freedom parallel robot as examples, the distribution of this index is analyzed. The results illustrate the impact of limb components on energy transmission in parallel robot execution systems. This index can be used to energy transmission analysis and the design of parallel equipments with high energy efficiency execution systems.

\section{energy efficiency analysis, parallel robot, power equation, mechanism execution system}

doi: $10.1360 /$ N092018-00156 